\title{
Influence of the injected beam parameters on the capture efficiency of an electron cyclotron resonance based charge breeder
}

\author{
A. Galatà \\ INFN-Laboratori Nazionali di Legnaro, Viale dell'Università 2, 35020 Legnaro, Padova, Italy \\ D. Mascali, G. Torrisi, L. Neri, and L. Celona \\ INFN-Laboratori Nazionali del Sud, Via S. Sofia 62, 95123 Catania, Italy \\ J. Angot \\ LPSC, Université Grenoble-Alpes, CNRS/IN2P3, 53 avenue des Martyrs, \\ 38026 Grenoble Cedex, France \\ (Received 9 March 2017; published 13 June 2017)
}

\begin{abstract}
Electron cyclotron resonance ion sources based charge breeders (ECR-CB) are fundamental devices for Isotope Separation On Line (ISOL) facilities aiming at postaccelerating radioactive ion beams (RIBs). Presently, low intensity RIBs do not allow a conventional tuning of the ECR-CB: as a consequence, it has to be set with a stable 1+ pilot beam first, switching then to the radioactive one without changing any parameter; this procedure is usually called "blind tuning." Besides having different masses, pilot and radioactive beams can also differ in terms of the rms transverse emittance $\epsilon_{\mathrm{rms}}$ and/or longitudinal energy spread $\Delta E$, so the choice of a given pilot beam can determine the overall performances of the final breeding stage. This paper shows a numerical study of how the capture efficiency of the PHOENIX charge breeder is affected by the aforementioned beam paramaters: the analysis reveals the two-step nature of the process, highlighting the role of the injection optics and the plasma capture capability in the overall performances of this device. The simulations predict highest efficiency for $\epsilon_{\mathrm{rms}}<5 \pi \mathrm{mm} \mathrm{mrad}$ and $\Delta E<5 \mathrm{eV}$ in a optimum energy range between 2 and $6 \mathrm{eV}$, thus giving important information on the possibility of blindly tuning an ECR-CB. No isotopical effects were observed, while it clearly came out the necessity to improve the $1+$ beam characteristics with a rf beam cooler prior to the injection into an ECR-CB.
\end{abstract}

DOI: 10.1103/PhysRevAccelBeams.20.063401

\section{INTRODUCTION}

The European road map of nuclear physics is now going through the development of second generation facilities pointing to EURopean ISOL radioactive ion beam facility (EURISOL) [1]: presently, three main projects are ongoing in different Laboratories, High Intensity and Energy-Isotope Separator On Line DEvice (ISOLDE) at CERN [2], Système de Production d'Ions Radioactifs Accélérés en Ligne 2 (SPIRAL2) at Grand accélérateur national d'ions lourds [3], and Selective Production of Exotic Species (SPES) at Istituto Nazionale di Fisica Nucleare-Legnaro National Laboratories (INFN-LNL) [4]. In most of the above mentioned facilities the $1+$ radioactive ion beam (RIB), produced in the so-called target-ion-source system, will be injected in a charge breeder to boost its charge state: this will be accomplished by adopting either electron beam ion sources (EBIS) [5] or electron cyclotron resonance ion sources (ECRIS) [6].

\footnotetext{
*alessio.galata@lnl.infn.it
}

Published by the American Physical Society under the terms of the Creative Commons Attribution 4.0 International license. Further distribution of this work must maintain attribution to the author(s) and the published article's title, journal citation, and DOI.
Both techniques were compared during the Sixth Framework Programme (FP6) [7], in view of the performances expected for EURISOL, and were found to be complementary: among the two, the ECRIS-based (ECR-CB), adopted at SPES [8], is usually chosen for the high acceptance in terms of injected current, the reliability, the possibility to operate both in continuous and pulsed mode, and the high charge states produced. The low intensity RIBs, presently injected in an ECR-CB, do not allow a real tuning of this device: as a consequence, it has to be set with a stable beam first, switching then to the radioactive one, having usually a different mass, without changing any parameter (injection optics included); this technique is known as "blind tuning" [9]. By applying this procedure, an important question arises: what happens to the charge breeder capture efficiency if the stable and radioactive beams have not the same properties? Or, equivalently, to what extent can an ECR-CB be blindly tuned? This paper tries to answer this question: a numerical code describing the charge breeding process is available at INFN, and has been used to analyze the effect of different beam parameters on the charge breeding efficiency: in particular, the transverse beam emittance, the longitudinal energy spread and the mass of the injected ions. The results and the following data analysis will be shown throughout the paper. 
TABLE I. Parameters used for the simulation of charge breeding inside the PHOENIX ECR-CB.

\begin{tabular}{lc}
\hline \hline Plasma parameters & \\
\hline Species & Oxygen \\
Average charge & 3 \\
Electron density $\left(\mathrm{m}^{-3}\right)$ & $1.96 \times 10^{17}$ \\
Warm electron temperature $(\mathrm{keV})$ & 1 \\
Ion temperature $(\mathrm{eV})$ & 0.3 \\
\hline \hline
\end{tabular}

\section{SIMULATIONS DESCRIPTION}

The applied simulation code has been extensively described elsewhere [10]: it basically simulates the ion beam slowing down and capture by a magnetized plasma, as a consequence of the long range Coulomb collisions with plasma ions. Once inside the plasma, injected ions are subject to a huge number of small angle elastic collisions, leading to thermal equilibrium with plasma ions. Such a process is numerically implemented by using the Langevin formalism [11,12], in a 3D "forward difference" integration scheme. The validity of the code was demonstrated by reproducing two experimental results obtained at the Laboratoire de Physique Subatomique et de Cosmologie (LPSC) test bench with the PHOENIX ECR-CB [13], in particular, with sodium [14] and rubidium ions [15]. The results of the latter simulations are taken as reference for the analysis presented in this paper: in particular, the charge breeding of ${ }^{85} \mathrm{Rb}^{1+}$ ions, injected at $20 \mathrm{keV}$ with $\epsilon_{\mathrm{rms}}=2 \pi \mathrm{mm} \operatorname{mrad}(\alpha=2.14, \beta=0.56)$ and $\Delta E=2 \mathrm{eV}$, by a plasma whose characteristics are shown in Table I. It is necessary to point out that the numerically deduced plasma parameters are in good agreement with an experimental evaluation carried out on the LPSC test bench [16]. The reported transverse emittance and associated Twiss parameters were measured upstream the PHOENIX booster in the LPSC test bench (characterization point), while the value of longitudinal energy spread is typical of the surface ionization sources used to produce ${ }^{85} \mathrm{Rb}^{1+}$ beams [17]. For the calculations described in [10] and in the present paper, particles start at the position corresponding to the maximum of the magnetic field at injection, where the potential is assumed to be equal to the plasma potential $V_{P}$ : initial spatial and velocity distributions were obtained by simulating with the tracking code SIMION [18] the beam transport from the characterization point to this location (see Fig. 1), considering the actual geometry of the LPSC beam line (a double Einzel lens focuses the beam into the charge breeder), and including the fringing field of the magnetic trap.

We focused our attention on the capture efficiency of ${ }^{85} \mathrm{Rb}^{1+}$ ions: experimentally, this parameter is defined as

$$
\epsilon_{c b}=\frac{1}{I_{1+}} \sum_{i=1}^{q_{\max }} \frac{I_{i+}}{i+}
$$

where $I_{1+}$ is the $1+$ beam injected current of a given species, and $I_{i+}$ is the current of the different charge states of the same species extracted from the charge breeder. Usually, $\epsilon_{c b}$ is measured as a function of the injection energy, defined as $E_{\text {inj }}=e\left(V_{1+}-V_{C B}\right)$, where $V_{1+}$ is the $1+$ source extraction potential, $V_{C B}$ is the charge breeder potential, and $e$ the elementary charge. This parameter is optimized by decreasing $V_{\mathrm{CB}}$ keeping $V_{1+}$ constant: this leads to the well-known parameter $\Delta V$, shown in all the experimental efficiency curves.

The plot of the ${ }^{85} \mathrm{Rb}^{1+}$ capture efficiency for the reference case is shown in Fig. 2: it reaches the highest values in a narrow energy range between 2 and $6 \mathrm{eV}$ (the optimum range from now on), where it shows a maximum, and then decreases quite quickly as soon as the energy increases. The trend can be very well fitted by a Gaussian curve of the type $f\left(E_{\text {inj }}\right)=a_{1} \exp \left\{-\left[\left(E_{\text {inj }}-b_{1}\right) / c_{1}\right]^{2}\right\}$ having, in the specific case, the following parameters: $a_{1}=49.17, b_{1}=4.82 \mathrm{eV}$, and $c_{1}=5.73 \mathrm{eV}$. It is important to point out that, for the present numerical simulations,

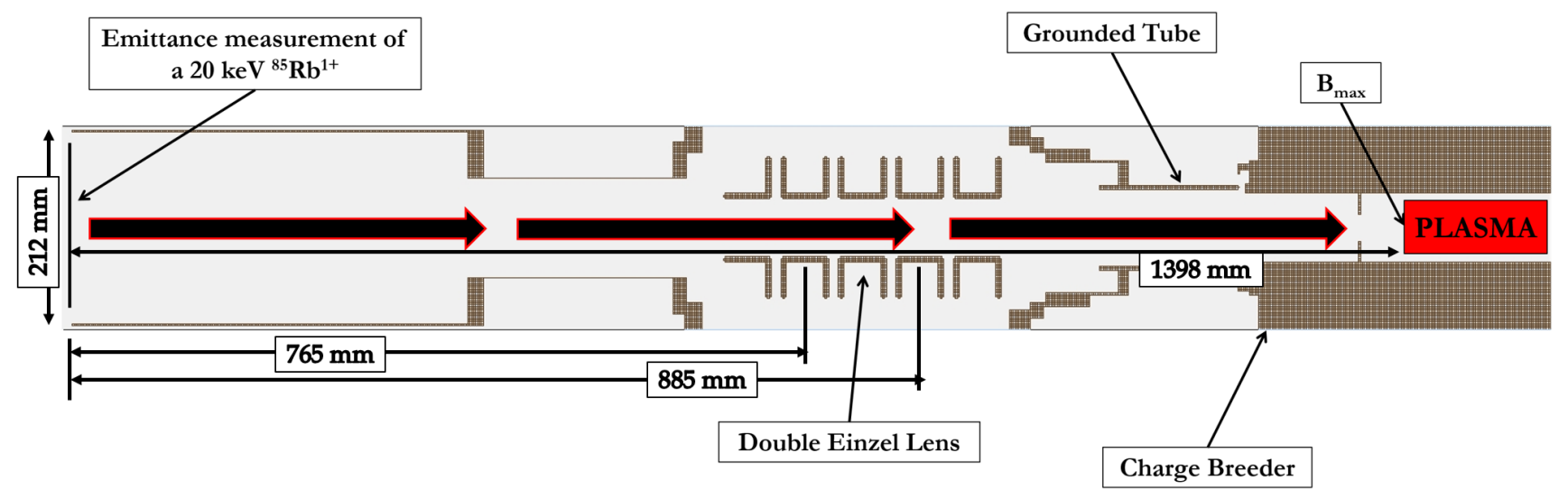

FIG. 1. Geometry used to simulate the injection of a ${ }^{85} \mathrm{Rb}^{1+}$ into the PHOENIX ECR-CB with the tracking code sIMION. Particles start at the characterization point (on the left in the figure), then, spatial and velocity distribution are obtained at the position corresponding to the maximum of the magnetic field at injection $\left(\mathrm{B}_{\max }\right)$. Cylindrical symmetry must be considered. 


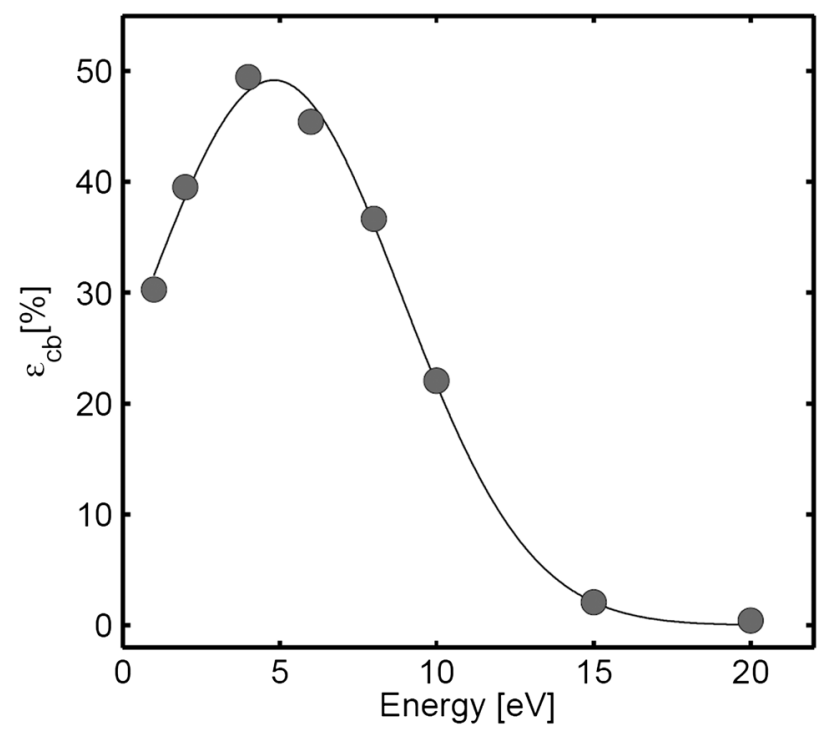

FIG. 2. ${ }^{85} \mathrm{Rb}^{1+}$ ions capture as a function of the injection energy inside the charge breeder for the reference simulations. The numerical results are very well fitted by a Gaussian curve.

the injection energy is defined as $E_{\mathrm{inj}}=e\left(V_{1+}-V_{P}\right)$ [10]: considering that $V_{P}>V_{\mathrm{CB}}, E_{\mathrm{inj}}$ is numerically lower compared to what is obtained experimentally. During the experiments, the best performances were obtained for $\Delta V_{\text {opt }}=V_{\mathrm{CB}}-V_{1+}=-12 \mathrm{~V}$, so an estimate for the plasma potential could be $V_{P}=6-8 \mathrm{~V}$. In the next sections it will be shown how the curve in Fig. 2 is modified by changing the beam parameters.

\section{RESULTS}

The first part of the analysis concerned the influence of the beam rms transverse emittance $\epsilon_{\text {rms }}$ on the capture efficiency: to this purpose, further simulations were carried out giving to this parameter the values $\epsilon_{\mathrm{rms}}=1,2,5$ and $10 \pi \mathrm{mmmrad}$ (typical of the surface and plasma ion sources employed in the ISOL facilities), keeping the same Twiss parameters mentioned in the previous section. The obtained capture efficiencies are compared in Fig. 3: we can observe that for the lowest values of the transverse emittance (including the reference case) the efficiency does not change appreciably, reaching the same (numerically) maximum for the same injection energy. When the transverse emittance increases the situation changes: the efficiency decreases by about $40 \%$ in the optimum range, and the maximum of the curve seems to shift towards higher energies. In view of a blind switch to a beam with a worse emittance, this translates in the risk of not only getting a lower efficiency but also of not reaching the highest possible efficiency, due to a mismatch in the optimum injection energy.

The second part of the analysis regarded the influence of the beam longitudinal energy spread, considering the values $0,2,5$, and $10 \mathrm{eV}$ : results are shown in Fig. 4.

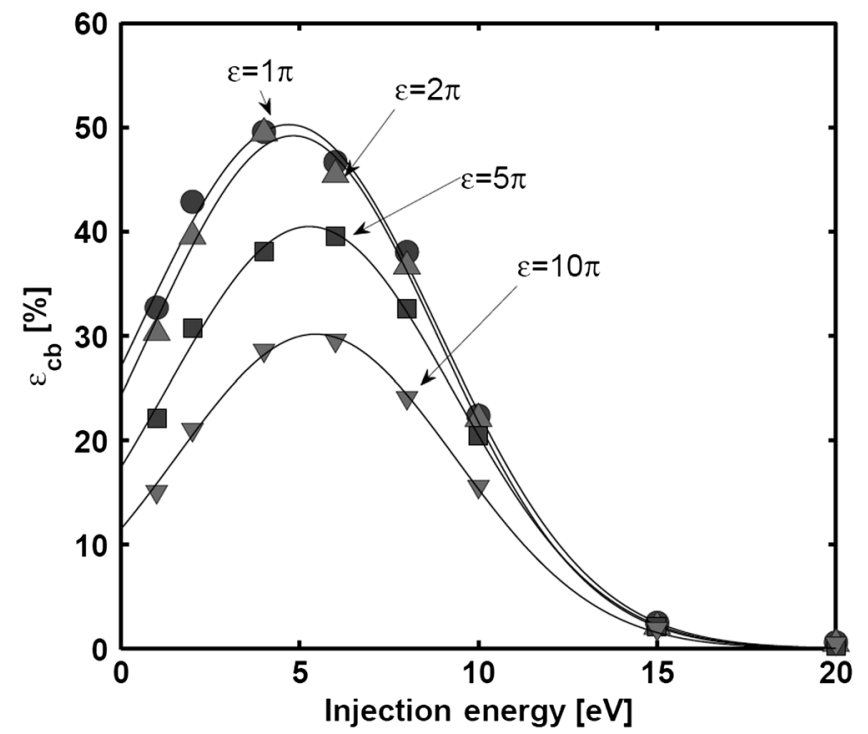

FIG. 3. ${ }^{85} \mathrm{Rb}^{1+}$ ions capture as a function of the injection energy inside the charge breeder for different values of the injected beam rms transverse emittance. The reference curve corresponds to $\epsilon_{\mathrm{rms}}=2 \pi \mathrm{mm}$ mrad. A Gaussian fit is superimposed to each numerical curve.

It can be observed that the influence of this parameter on the capture efficiency is stronger: for the lowest values, typical of the surface ionization $1+$ sources, there are not remarkable differences between the two curves but, as soon as the longitudinal energy spread increases to values typical of plasma 1+ ion sources [17], the efficiency drops immediately and the curves become more flat. In this condition, the efficiency is reduced of a factor up to more

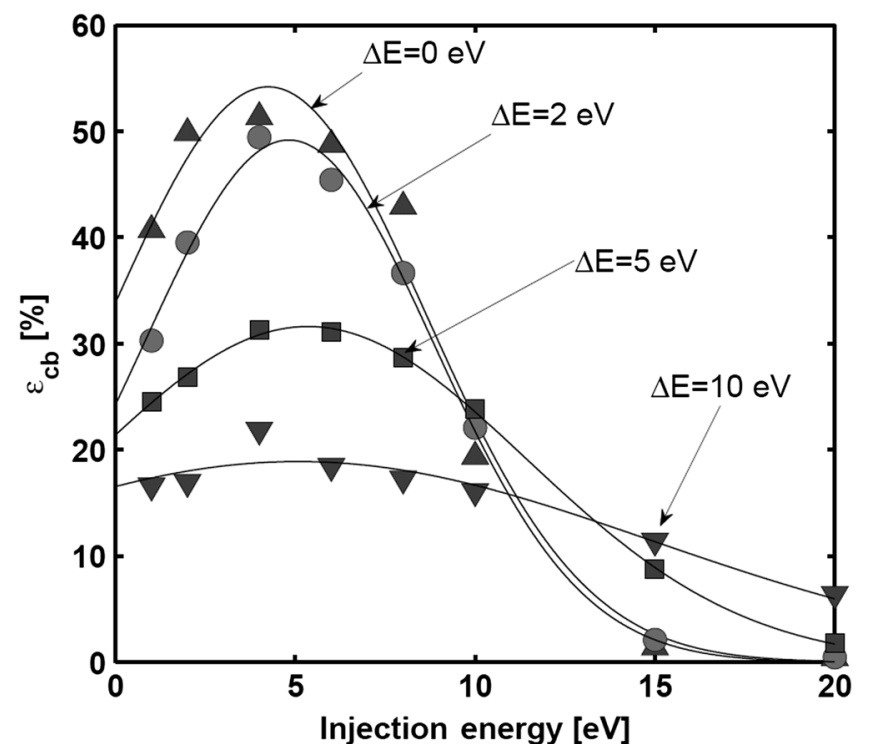

FIG. 4. ${ }^{85} \mathrm{Rb}^{1+}$ ions captured as a function of the injection energy inside the charge breeder for different values of the injected beam longitudinal energy spread. The reference curve corresponds to $2 \mathrm{eV}$. A Gaussian fit is superimposed on each numerical curve. 


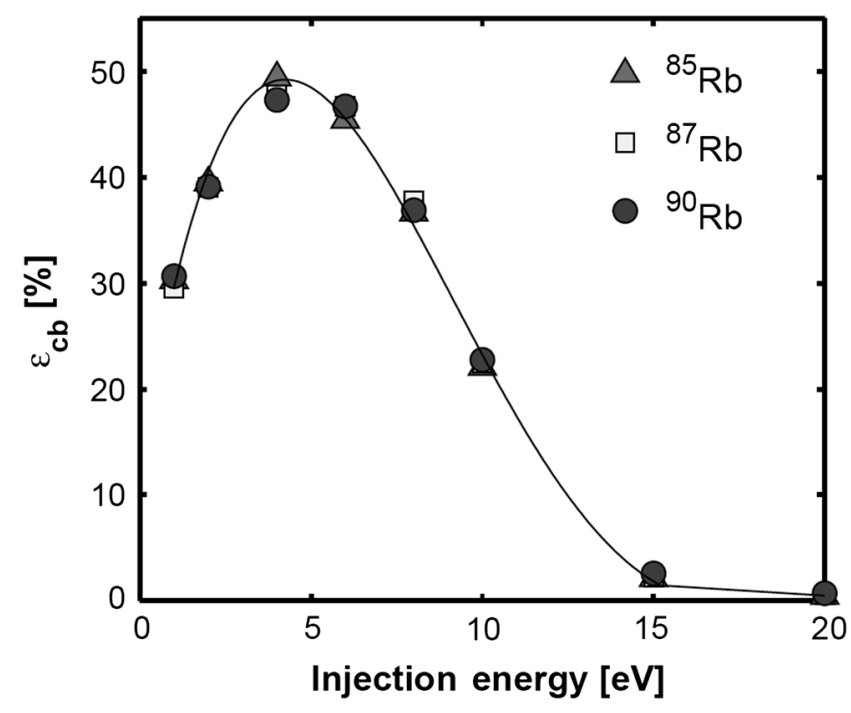

FIG. 5. Ion captured as a function of the injection energy inside the charge breeder for different Rubidium isotopes and beam injection energy. The reference curve corresponds to ${ }^{85} \mathrm{Rb}$. A Gaussian fit is superimposed on each numerical curve.

than 2, and the injection energy does not have a strong influence on the (low) capture efficiency. A positive aspect is that all the simulations show their maxima for the same injection energy: from the point of view of the blind tuning, this means that switching to a beam with a worse energy spread would imply a lower efficiency, anyway obtaining the maximum possible value.

Finally, in view of the future operation at SPES, we simulated the injection of the three different rubidium isotopes, ${ }^{85} \mathrm{Rb},{ }^{87} \mathrm{Rb}$, and ${ }^{90} \mathrm{Rb}$, with the parameters shown in Table I. The first two are stable isotopes, and the last is one of the main radioactive species which is expected to be produced at SPES: this physics case reflects the experimental condition in which the SPES charge breeder will be tuned with stable $\mathrm{Rb}^{1+}$ ions, to finally charge breed radioactive ${ }^{90} \mathrm{Rb}^{1+}$ ions. The capture efficiencies are compared in Fig. 5; under the hypothesis of constant beam parameters, switching from stables to radioactive rubidium ions will result in the same efficiency, with the maximum at the same injection energy. This means that, in the specific case, the blind tuning would be effective.

\section{DISCUSSION}

The capture efficiency of a charge breeder $\epsilon_{c b}$, defined in Eq. (1) and plotted in Figs. 3-5, can be expressed, from a physical point of view, as

$$
\epsilon_{c b}=\epsilon_{\text {inj }} \cdot \epsilon_{\text {capt }},
$$

where the first term on the right is the injection efficiency, while the second is the plasma capture efficiency. In fact, during the injection process, $1+$ ions are decelerated to very low energies by the charge breeder potential, and in its proximity they start feeling the influence of its growing axial magnetic fringing field. As in the case of magnetic trapping, it exerts a force on the $1+$ ions directed toward the low field region, that is away from the charge breeder. This finally leads to ion reflections, so the term $\epsilon_{\text {inj }}$ takes into account for the $1+$ ions that are "transmitted" inside the charge breeder. Considering that the axial magnetic force is proportional to the transversal component of ions energy (velocity), $\epsilon_{\text {inj }}$ could be directly correlated to beam transverse emittance. Only the transmitted ions effectively interact with the plasma through the process described in Sec. II, and the term $\epsilon_{\text {capt }}$ takes into account their capture efficiency: the interaction with the plasma depends on the ions energy through their velocity, so $\epsilon_{\text {capt }}$ could be correlated to the beam longitudinal energy spread.

In this view, it is interesting to try to distinguish the relative effects of the two previously defined quantities on $\epsilon_{c b}$ : to this purpose, the ion capture efficiency $\epsilon_{c b}$, shown in Figs. 3 and 4, has been recalculated to give $\epsilon_{\text {capt }}$, that is net of reflections. The obtained "normalized" capture efficiencies are shown in Figs. 6 and 7: in both cases the maxima shifted towards very low energies, meaning that the optimum value, visible in Figs. 3-5, is a consequence of the injection process and not of the interaction with the plasma. Moreover, the highest values obtained exceed the ones showed in Figs. 3 and 4, so reflections in a way reduce the real performances of a charge breeder. Apart from these similarities, the normalization procedure had a different effect on the two sets of curves previously shown: in Fig. 6 we can see that the differences are drastically reduced, especially in the optimum range. The transverse emittance has a limited effect on $\epsilon_{\text {capt }}$, confirming that this parameter

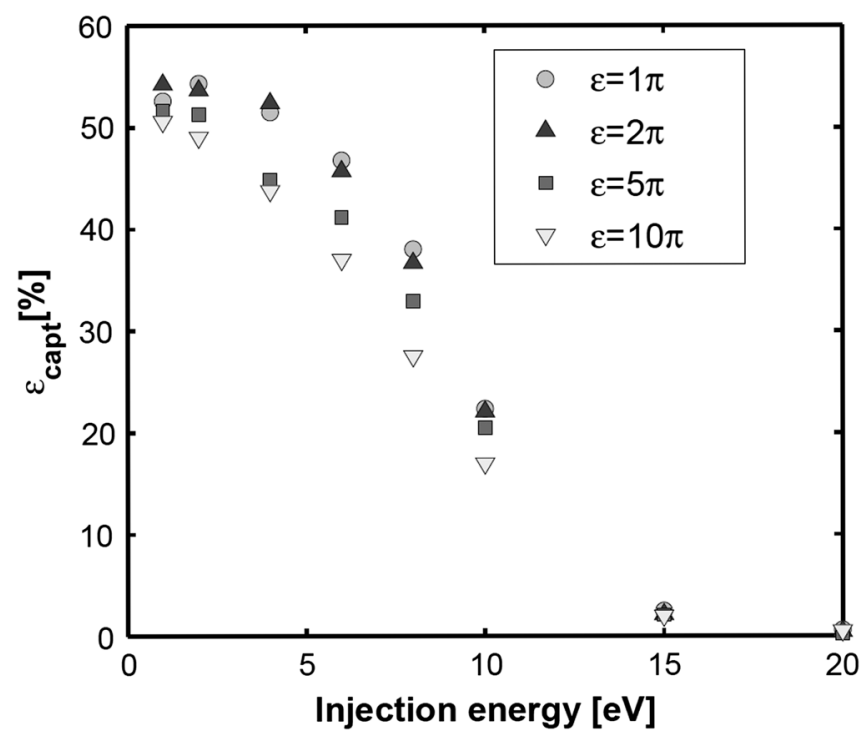

FIG. 6. Normalized ions captured as a function of the injection energy inside the charge breeder for different values of the injected beam rms transverse emittance. 


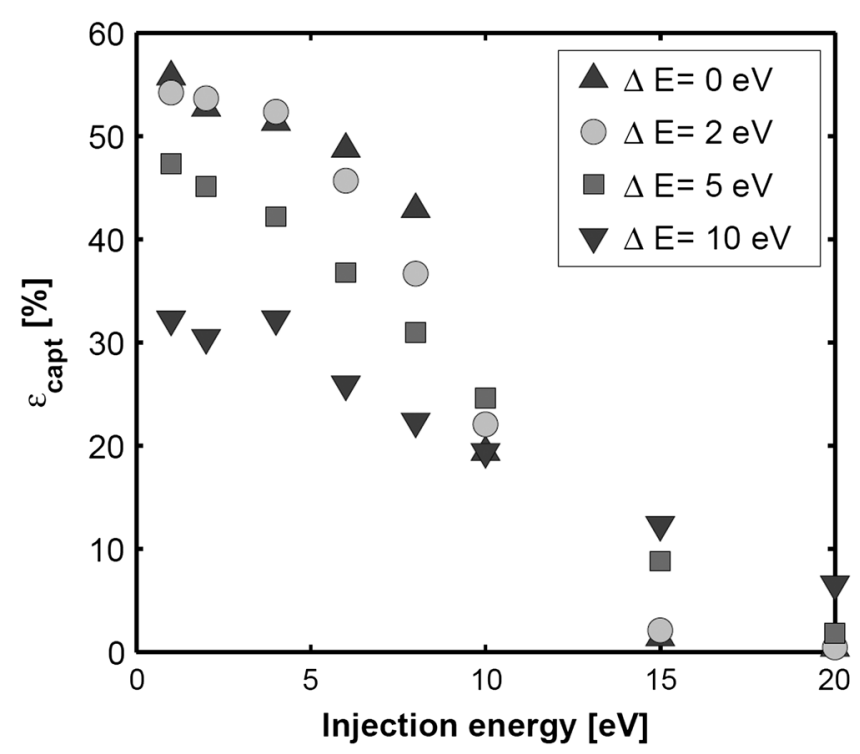

FIG. 7. Normalized ions captured as a function of the injection energy inside the charge breeder for different values of the injected beam longitudinal energy spread.

basically affects $\epsilon_{\text {inj. }}$. If we think about transverse emittances as different velocity distributions for a given beam energy, the results shown in Fig. 6 are consistent with theory, which foresees ion thermalization is reached whatever the initial particle distribution function [19]. The situation is different in Fig. 7: even without considering reflected particles, the effect of the longitudinal energy spread is still evident, especially in the optimum range. Here, in fact, for values above $2 \mathrm{eV}$, the longitudinal energy spread is higher than the injection energy, and we observe the effect of the interaction between a plasma and a beam of nearly uniform energy. Finally, at high energy spreads the trend of the curves changes, and numerical results show a nearly linear dependence of $\epsilon_{\text {capt }}$ from $E_{\text {inj }}$.

\section{CONCLUSION AND PERSPECTIVES}

This paper presented an analysis of the influence of transverse emittance and longitudinal energy spread on the efficiency on an ECR-CB, made by numerical simulations. By expressing the charge breeding efficiency as in Eq. (2), simulations revealed that the injection efficiency is mainly affected by the transverse emittance, due to reflection by the magnetic fringing field, while the capture efficiency is directly correlated to the beam longitudinal energy spread. Figures 3 and 4 tell us that a blind tuning with beams of different transverse emittance or longitudinal energy spread seems feasible, with the only risk of not preserving the maximum efficiency when injecting beams with a high $\epsilon_{\mathrm{rms}}$. Finally, under the hypothesis of constant beam parameters, stable rubidium $1+$ ions are the best candidates to set the ECR-CB for blind tuning of ${ }^{90} \mathrm{Rb}^{1+}$ ions at SPES.
In general, results tell us that a good $1+$ beam quality is an essential ingredient for a high charge breeding efficiency: this is typical for $1+$ beams produced by surface ionization sources (essentially elements of the first group in the periodic table), while generally this is not the case for plasma ionization sources. Because of the variety of radioactive species produced, ISOL facilities foresee the use of both kinds of sources, so for optimum operation it could be necessary to properly manipulate the $1+$ beam, in order to improve its characteristics before delivering it to the breeding stage. This will be the case of the SPES project, where a complete charge breeding beam line, equipped with a radio frequency quadrupole (RFQ) beam cooler [20], will be put into operation starting in 2018, allowing us the verifications of the simulations results presented in this paper. By comparing Figs. 3 and 6, it can be seen that the charge breeding efficiency could be further improved by limiting beam reflections: with this in mind, a possibility (to be verified) could be the injection of a beam with a small divergence (tansverse velocity component $v_{\perp}$ ), so as to reduce the reflecting magnetic force $\vec{F}=-\frac{m v_{\perp}^{2}}{2 B} \nabla B$.

The numerical code developed in these last years is providing valuable results for the optimization of existing facilities and for addressing the design of future ones. Among others, the Plasmas for Advancements in Nuclear Decays Observationand as x-Ray source for Archaeometry (PANDORA) project [21] is now starting at INFN: it is based on a new use of ECR plasmas, which become an environment for measuring, for the first time, nuclear decay rates of different radionuclides as a function of their ionization state. Among the methods used to introduce such radionuclides inside the trap, the charge breeding technique has been judged as the most efficient, and must be optimized in case of especially light elements such as ${ }^{7} \mathrm{Be}$ (that will be the first physics case due to the relevant role played in nuclear astrophysics).

[1] http://www.eurisol.org/.

[2] A. Herlert and Y. Kadi, The HIE-ISOLDE Project, J. Phys. Conf. Ser. 312, 052010 (2011).

[3] S. Gales, GANIL-SPIRAL2: A new era, AIP Conf. Proc. 1355, 36 (2011).

[4] G. Bisoffi et al., Progress in the design and construction of SPES at INFN-LNL, Nucl. Instrum. Methods Phys. Res., Sect. B 376, 402 (2016).

[5] E. D. Donets, Historical review of electron beam ion sources (invited), Rev. Sci. Instrum. 69, 614 (1998).

[6] R. Geller, Electron Cyclotron Resonance Ion Source and ECR Plasmas (IOP, Bristol, England, 1996).

[7] P. Delahaye, O. Kester, C. Barton, T. Lamy, M. Marie-Jeanne, and F. Wenander, Evaluation of charge breeding options for EURISOL, Eur. Phys. J. A 46, 421 (2010). 
[8] A. Galatà et al., Status of the SPES-charge breeder (SPESCB) and its beam line at INFN-LNL, Nucl. Instrum. Methods Phys. Res., Sect. B 376, 329 (2016).

[9] T. Lamy, J. Angot, and T. Thuillier, European research activities on charge state breeding related to radioactive ion beam facilities (invited), Rev. Sci. Instrum. 79, 02 A909 (2008).

[10] A Galatà, D Mascali, L Neri, and L Celona, A new numerical description of the interaction of an ion beam with a magnetized plasma in an ECR-based charge breeding device, Plasma Sources Sci. Technol. 25, 045007 (2016).

[11] S. Chandrasekhar, Stochastic Problems in Physics and Astronomy, Rev. Mod. Phys. 15, 1 (1943).

[12] W. M. Manheimer, M. Lampe, and G. Joyce, Langevin Representation of Coulomb Collisions in PIC Simulations, J. Comput. Phys. 138, 563 (1997).

[13] T. Lamy et al., Charge State breeding applications with the ECR PHOENIX source: From low to high current production, Rev. Sci. Instrum. 73, 717 (2002).

[14] O. Tarvainen, H. Koivisto, A. Galatà, J. Angot, T. Lamy, T. Thuillier, P. Delahaye, L. Maunoury, D. Mascali, and L. Neri, Diagnostic of a charge breeder electron cyclotron resonance ion source helium plasma with the injection of ${ }^{23} \mathrm{Na}^{1+}$ ions, Phys. Rev. Accel. Beams 19, 053402 (2016).

[15] A. Galatà, D. Mascali, L. Neri, G. Torrisi, and L. Celona, A three-dimensional numerical modelling of the PHOENIXSPES charge breeder based on the Langevin formalism, Rev. Sci. Instrum. 87, $02 B 507$ (2016).

[16] O. Tarvainen et al., Injected $1+$ ion beam as a diagnostic tool of charge breeder ECR ion source plasmas, Plasma Sources Sci. Technol. 24, 035014 (2015).

[17] B. Wolf, Handbook of Ion Sources (CRC Press, Boca Raton, FL, 1995).

[18] simion.com, Scientific Instrument Services, Inc. (SIS).

[19] L. C. Woods, Principles of Magnetoplasma Dynamics (Oxford Science Publication, Oxford, England, 1987).

[20] M. Maggiore et al., Status of the RFQ Beam Cooler for SPES project at LNL, Eur. Phys. J. Web Conf. 66, 11024 (2014).

[21] D. Mascali et al., PANDORA a New Facility for Interdisciplinary In-Plasma Physics, http://arxiv.org/abs/1703 .00479 . 